\title{
Lysyl oxidase secreted by tumour endothelial cells promotes angiogenesis and metastasis
}

\author{
T Osawa ${ }^{1,2}$, N Ohga ${ }^{1}$, K Akiyama ${ }^{1}$, Y Hida $^{3}$, K Kitayama $^{1}$, T Kawamoto $^{1}, \mathrm{~K} \mathrm{Yamamoto}^{1}$, N Maishi ${ }^{1}$, \\ M Kondoh ${ }^{1}$, Y Onodera ${ }^{1}$, M Fujie ${ }^{4}$, N Shinohara ${ }^{2}$, K Nonomura ${ }^{2}$, M Shindoh ${ }^{5}$ and K Hida*,1 \\ ${ }^{1}$ Department of Vascular Biology, Graduate School of Dental Medicine, Hokkaido University, Sapporo, Japan; ${ }^{2}$ Department of \\ Urology, Graduate School of Medicine, Hokkaido University, Sapporo, Japan; ${ }^{3}$ Department of Cardiovascular and Thoracic \\ Surgery, Graduate School of Medicine, Hokkaido University, Sapporo, Japan; ${ }^{4}$ DNA Sequencing Center Section, Okinawa Institute \\ of Science and Technology Promotion Corporation, Onna, Okinawa, Japan and ${ }^{5}$ Department of Oral Pathology and Biology, \\ Graduate School of Dental Medicine, Hokkaido University, Sapporo, Japan
}

Background: Molecules that are highly expressed in tumour endothelial cells (TECs) may be candidates for specifically targeting TECs. Using DNA microarray analysis, we found that the lysyl oxidase (LOX) gene was upregulated in TECs compared with its expression in normal endothelial cells (NECs). LOX is an enzyme that enhances invasion and metastasis of tumour cells. However, there are no reports on the function of LOX in isolated TECs.

Methods: TECs and NECs were isolated to investigate LOX function in TECs. LOX inhibition of in vivo tumour growth was also assessed using $\beta$-aminopropionitrile (BAPN).

Results: LOX expression was higher in TECs than in NECs. LOX knockdown inhibited cell migration and tube formation by TECs, which was associated with decreased phosphorylation of focal adhesion kinase (Tyr 397). Immunostaining showed high LOX expression in human tumour vessels in vivo. Tumour angiogenesis and micrometastasis were inhibited by BAPN in an in vivo tumour model.

Conclusion: LOX may be a TEC marker and a possible therapeutic target for novel antiangiogenic therapy.

Solid tumours are dependent on angiogenesis for their growth beyond 1-2 mm in size (Folkman, 1971) and for tumour metastasis (Folkman, 1995). Therefore, targeting tumour vessels can inhibit tumour growth and metastasis.

Antiangiogenic therapy is an effective strategy for several types of cancer (Randall and Monk, 2010). Recent success with bevacizumab, a humanised monoclonal antibody against vascular endothelial growth factor (VEGF), in prolonging the lives of patients with metastatic colon cancer established the validity of antiangiogenic therapy (McCarthy, 2003). However, several side effects have been reported with this antiangiogenic therapy. Because VEGF has an important role in normal physiological processes, such as stabilizing damaged endothelium and wound healing, VEGF inhibition may result in undesirable toxicity in normal tissues.
It was recently reported that tumour endothelial cells (TECs) differed from normal endothelial cells (NECs) in that TECs expressed specific markers, including tumour endothelial markers (TEMs) (St Croix et al, 2000), epithelial growth factor receptor (EGFR) (Amin et al, 2006) and several other molecules (Maishi et al, 2012; Osawa et al, 2012; Yamamoto et al, 2012). TECs also exhibit different biological behaviours; for example, they grow and migrate faster than NECs (Matsuda et al, 2010).

We previously reported that TECs exhibited different responses to several drugs compared with NECs (Amin et al, 2006; Ohga et al, 2009; Tsuchiya et al, 2010; Muraki et al, 2011). TECs are also more resistant to chemotherapeutic drugs (Bussolati et al, 2003; Akiyama et al, 2012; Ohga et al, 2012). Furthermore, TECs harbour certain cytogenetic abnormalities (Hida et al, 2004; Hida and Klagsbrun, 2005; Akino et al, 2009). Thus, to reduce the clinical 
adverse effects when using a vascular targeting approach, it is critical to identify a therapeutic target specifically expressed in TECs.

Using DNA microarray analysis, we identified several markers highly expressed in TECs compared with that in NECs (Yamamoto et al, 2012). In this study, we focused on lysyl oxidase (LOX) upregulated approximately 190 -fold in TECs compared with that in NECs.

LOX is a copper-dependent amine oxidase that covalently crosslinks collagen and elastin in the extracellular matrix and maintains tissue integrity necessary for normal connective tissue function (Trackman et al, 1992; Panchenko et al, 1996; Lucero and Kagan, 2006). Several studies have shown that LOX was expressed in various types of tumour cells and had a role in tumour progression and metastasis (Kirschmann et al, 2002; Erler et al, 2006). However, a role for LOX in TECs has not been examined. Therefore, we examined LOX expression and its function in TECs in vitro and in vivo.

\section{MATERIALS AND METHODS}

Cell lines and culture conditions. A375SM cells, a supermetastatic human malignant melanoma cell line, were a gift from Dr Isaiah J Fidler (MD Anderson Cancer Center, Houston, TX, USA). MDA-MB-231 cells were purchased from the ATCC (Manassas, VA, USA). Cells were cultured in minimum essential medium (Gibco, Grand Island, NY, USA) as described previously (Osawa et al, 2012).

Isolation of TECs and NECs. All procedures for animal experiments were approved by the local animal research authorities, and animal care was carried out in accordance with institutional guidelines. Mouse TECs (mTECs) and NECs (mNECs) were isolated as previously described with some modifications (Osawa et al, 2012). Diphtheria toxin (DT; $500 \mathrm{ng} \mathrm{m}^{-1}$; Calbiochem, San Diego, CA, USA) was added to mTEC subcultures to kill any human tumour cells (DT was also added to mNEC subcultures for technical consistency). Using an anti-human CD31 antibody, human TECs (hTECs) and NECs (hNECs) were isolated from excised renal cell carcinomas and normal renal tissues of six patients at Hokkaido University Hospital. These protocols were approved by the Ethics Committee of Hokkaido University, and written informed consent was obtained from each patient before surgery. ECs were cultured as described above, except that no DT was added.

Flow cytometry. Labelled cells were analysed with a FACS Aria II (Becton Dickinson, San Jose, CA, USA), and data were analysed using the FlowJo software (Treestar, Ashland, OR, USA). The following antibodies were used: rat, anti-mouse CD105 (BD Pharmingen, San Diego, CA, USA); rat, anti-mouse CD144 (BD Pharmingen); APC-conjugated rat, anti-mouse CD11b (BioLegend, San Diego, CA, USA); APC-conjugated rat, anti-mouse CD45 (BioLegend); mouse, anti-human CD105 (BD Pharmingen); PEconjugated mouse, anti-human CD45 (BD Pharmingen); Alexa Fluor 488 goat, anti-rat IgG (Invitrogen, Tokyo, Japan); and Alexa Fluor 488 goat, anti-mouse IgG. FITC-Bandeiraea simplicifolia lectin isolectin B4 (BS1-B4; Vector Laboratories, Burlingame, CA, USA) was used for mouse Ecs, and fluorescein-labelled ulex europaeus agglutinin I (UEA1-Letctin) (Vector Laboratories) was used for human ECs.

Reverse transcription and quantitative polymerase chain reaction. Total RNA was extracted from cells using an RNeasy Micro Kit (Qiagen, Valencia, CA, USA). Complementary DNA (cDNA) was synthesised using a ReverTra-Plus (Toyobo, Osaka, Japan) as described previously (Osawa et al, 2012). Relative quantification of target mRNA used SsoFast EvaGreen Supermix quantitative PCR detection (CFX 96 real-time PCR detection system, Bio-Rad, Hercules, CA, USA) for mouse ECs and SYBR Green Real-time PCR Master Mix-Plus (Bio-Rad) for human ECs (in triplicate) according to the manufacturer's protocols. qPCR amplification was performed at $95^{\circ} \mathrm{C}$ for $3 \mathrm{~min}$ and 45 cycles at $95^{\circ} \mathrm{C}$ for $10 \mathrm{~s}$ and $60^{\circ} \mathrm{C}$ for $30 \mathrm{~s}$. Data were analysed with the CFX manager software (Bio-Rad) (Osawa et al, 2012). Each experiment included four PCR reactions, and each experiment was repeated three times (Supplementary Figure 1).

Western blotting. Western blotting used antibodies specific for LOX (Abnova, Taipei, Taiwan), total focal adhesion kinase (FAK), phosphorylated FAK Tyr-397 (Cell Signaling Technology, Beverly, MA, USA), $\beta$-actin, GAPDH and an HRP-conjugated secondary antibody as described previously (Kawamoto et al, 2012). Densitometry readings were analysed using the NIH Image software (Bethesda, MD, USA).

Immunostaining. Tumour tissues were dissected from humanely killed mice. Human tissue samples were obtained from excised renal cell carcinoma and normal renal tissues of six patients at Hokkaido University Hospital. Frozen sections of excised tissue were prepared as described (Kurosu et al, 2011). Mouse sections were double stained with rat, anti-mouse CD31/Alexa Fluor 594 goat, anti-rat IgG and anti-LOX/Alexa Fluor 488 goat, anti-rabbit IgG (Invitrogen) to assess LOX co-localisation in ECs. TECs were fixed in cold methanol and immunostained with mouse, antihuman vinculin (Sigma Chemical Co., St Louis, MO, USA) and MFP488-phalloidin (MoBiTec, Gottingen, Germany). Human sections were double stained with mouse, anti-human CD31/Alexa Fluor 594 rat, anti-mouse IgG and anti-LOX/Alexa Fluor 488 goat, anti-rabbit IgG. All samples were counterstained with DAPI (Roche Diagnostics, Mannheim, Germany) and examined using an Olympus FluoView FV10i confocal microscope (Olympus, Tokyo, Japan).

LOX knockdown. LOX siRNA was transfected into cells using Hiperfect transfection reagent (Qiagen) according to the manufacturer's instructions. LOX siRNA was 5'-CUGGCGCCAGA CAAUCCAAUU- $3^{\prime}$ and control siRNA was a non-targeting control (Genolution Pharmaceuticals Inc., Seoul, South Korea).

LOX activity assay. To assess LOX activity in ECs, hydrogen peroxide levels in EC-conditioned media were determined using the LOX enzymatic inhibitor $\beta$-aminopropionitrile (BAPN; $500 \mu \mathrm{M}$; Sigma-Aldrich, St Louis, MO, USA) as described previously (Erler et al, 2009; Rodriguez et al, 2010) using a Vario Scan Flash plate reader (Thermo, Rockford, IL, USA). Assays were independently performed three times with similar results.

Random motility assay. mTECs random motility was monitored by tracking cell nuclei every $10 \mathrm{~min}$ for $7 \mathrm{~h}$ with an FV10i microscopic time-lapse imaging system (Olympus). Cell migration distances were quantified as described previously (Kawamoto et al, 2012).

Tube formation assays. Tube formation assays were performed with or without BAPN $(500 \mu \mathrm{M})$ using growth factor-reduced Matrigel (BD Biosciences, San Jose, CA, USA) as described previously (Osawa et al, 2012). Tube length was measured using the ImageJ software. Experimental results were recorded at three different time points. To investigate FAK involvement in TEC tube formation, assays were performed either with or without a FAK inhibitor $(0,5$ or $10 \mu \mathrm{M}$; Tocris Bioscience, Bristol, UK).

Cell proliferation assay. Cell proliferation was assessed with an MTS assay as described previously (Ohga et al, 2009). TECs were treated with control siRNA, LOX siRNA or BAPN $(0,500 \mu \mathrm{M})$ in $5 \%$ EBM2 for $24 \mathrm{~h}$. Each experiment was repeated three times with similar results. 
Tumour xenografts in mice. Pathogen-free six-week-old female $\mathrm{BALB} / \mathrm{c}$ nude mice (nu/nu) were obtained from Sankyo Laboratory Service Corporation (Tokyo, Japan) and randomly divided into two groups ( $n=3$ per group). A375SM cells $\left(2 \times 10^{6}\right.$ cells/ mouse) were subcutaneously inoculated into the right flanks of nude mice. Each mouse received a single intraperitoneal injection of BAPN (100 $\mathrm{mg} \mathrm{kg}^{-1}$; Sigma, St Louis, MO, USA) before tumour induction (day -1) and everyday thereafter for the duration of the experiment. The same dose of BAPN was used as described previously (Erler et al, 2006; Bondareva et al, 2009; Levental et al, 2009). Control mice were injected with vehicle (sterile distilled water). Tumour growth was monitored for 21 days. Body weights did not change in these groups throughout the experimental period.

Analysis of circulating tumour cell numbers. Peripheral blood was collected from each mouse in the control and BAPN-treated tumour groups ( $n=3$ per group) before humane killing. Mononuclear cells were isolated from mouse peripheral blood samples as described previously with modifications (Ohga et al, 2009). Peripheral blood mononuclear cells (PBMCs) were incubated with FITC-anti-human HLA to determine the numbers of circulating human tumour cells (CTCs). A minimum of 100000 cells per sample were analysed using a FACS Aria II.

Microvessel density and micrometastasis assessments in vivo. On day 21, mice were anaesthetised and tumours were resected. Frozen sections were prepared, and the microvessel density (MVD) of each CD31-stained tumour was determined in five randomly selected fields. The percentage of CD31-stained areas was analysed in five randomly selected fields using the ImageJ software. Tissue sections were stained with mouse monoclonal anti-human Ki-67 (DAKO) using Vulcan Red (Biocare Medical, Concord, CA, USA). Nuclei were counterstained with haematoxylin. Sections were examined with a light microscope (BX43; Olympus, Tokyo, Japan). The number of metastasised tumour colonies (>20 Ki67-positive cells) in the lung were determined for three randomly chosen sections. To identify cross-linked collagen fibres in the lung, lung sections were stained with Picrosirius Red (Polysciences, Warrington, PA, USA) and examined by phase contrast microscopy under polarised light for five randomly chosen sections.

Statistical analysis. Results are given as means \pm s.d. Group comparisons were made by Mann-Whitney $U$-test for continuous variables. Comparisons within groups at each time point were made by paired $t$-tests. $P<0.05$ was considered significant, and $P<0.01$ was considered highly significant.

\section{RESULTS}

Isolation and characterisation of mTECs and mNECs. mTECs were isolated from A375SM xenografts in nude mice. As a normal control, mNECs were isolated from mouse dermis. BS1-B4 lectin binding and CD31, CD105 and CD144 expression established that the isolated ECs were of high purity. mECs were negative for the monocyte marker CD11b and the hematopoietic marker CD45 (Figure 1A).

Because DT was used, which binds to human HB-EGF and can kill human cells, cultured mTECs were not contaminated with human tumour cells. Using RT-PCR, we confirmed that mECs did not express human HB-EGF.

LOX mRNA and protein expressions are upregulated in mTECs. LOX mRNA expression in mECs was assessed by qPCR. LOX mRNA expression was higher in mTECs than in mNECs (Figure 1B). In addition, western blotting showed that LOX protein expression was increased in mTECs compared with that in mNECs (Figure 1C and Supplementary Figure 2). LOX catalytic activity was also higher in the culture supernatants of mTECs compared with that in mNEC culture supernatants (Figure 1D), which suggested that LOX activity was significantly increased in mTECs.

LOX is expressed in mouse tumour blood vessels in vivo. To analyse the in vivo expression of LOX in TECs, fluorescent double immunostaining of an A375SM tumour xenograft in a mouse was performed using anti-LOX and anti-CD31 antibodies (Figure 2). LOX was predominantly expressed in tumour vessels and weakly expressed in normal vessels.

LOX knockdown in mTECs by siRNA. To investigate the role of LOX in mTECs, LOX expression in mTECs was silenced using LOX siRNA. LOX mRNA knockdown was confirmed at $24-72 \mathrm{~h}$ after transfection using qPCR (Figure $3 \mathrm{~A}$ ). LOX protein knockdown in mTECs was also confirmed (Figure 3B). In addition, LOX catalytic activity in mTECs was downregulated after LOX knockdown (Figure 3B).

LOX knockdown inhibits cell motility concomitant with morphological changes in mTECs. To assess the effects of LOX knockdown on the angiogenic phenotype of mTECs, we analysed random cell motility using time-lapse microscopy. Total movement distances were compared between control and LOX-knockdown mTECs. During the same period of time, LOX-knockdown mTECs moved a shorter distance $(136 \mu \mathrm{m})$ than the control mTECs (508 $\mu \mathrm{m}$; Figure 3C).

Cell morphology and cytoskeletal status are important for cell migration (Small, 1981). Control mTECs had spiked shapes with thin protrusions. In contrast, LOX knockdown and staining with phalloidin demonstrated that there were typical actin stress fibres throughout these mTECs. The ratio of cell length to cell width in mTECs decreased after LOX knockdown (Figure 3D).

Cell migration is coordinated by a complex of proteins that localise at sites of cell-matrix interactions or focal adhesions (Humphries et al, 2007). A key regulator of focal adhesions is the adaptor protein vinculin, which is a component of focal adhesion contacts and an anchor for actin filaments. Vinculin overexpression inhibits cell migration (Coll et al, 1995). Vinculin expression was increased in mTECs after LOX knockdown (Figure 3D), whereas control cells showed a thin 'mesenchymal cell-like shape' with minimal vinculin expression. These results suggested that LOX regulated cell morphology and migration through focal adhesion molecules in mTECs.

However, mTEC proliferation and viability were unaffected by LOX knockdown (Supplementary Figure 3).

LOX knockdown reduces phosphorylated FAK (Tyr 397) levels and inhibits tube formation. Previous studies showed that LOX had an important role in the migration and invasion of several cancer cell lines by activating FAK (Kirschmann et al, 2002; Erler et al, 2006; Levental et al, 2009). Because mTECs motility was possibly regulated by LOX, we examined FAK activation as a candidate signalling pathway regulated by LOX. Western blotting showed that phosphorylated FAK (Tyr 397) levels were reduced by LOX knockdown (Figure 4A), which suggested that LOX enhanced mTECs motility through FAK signalling. A FAK inhibitor reduced tube formation by mTECs (Figure 4B). In addition, LOX knockdown significantly reduced tube formation by mTECs (Figure 4C). These results suggested that LOX enhanced the pro-angiogenic phenotype of mTECs through FAK activation.

BAPN inhibits tumour angiogenesis and lung metastasis in vivo. BAPN irreversibly blocks the amine oxidase activity of LOX, and BAPN has been used to inhibit LOX activity (Wilmarth 
A
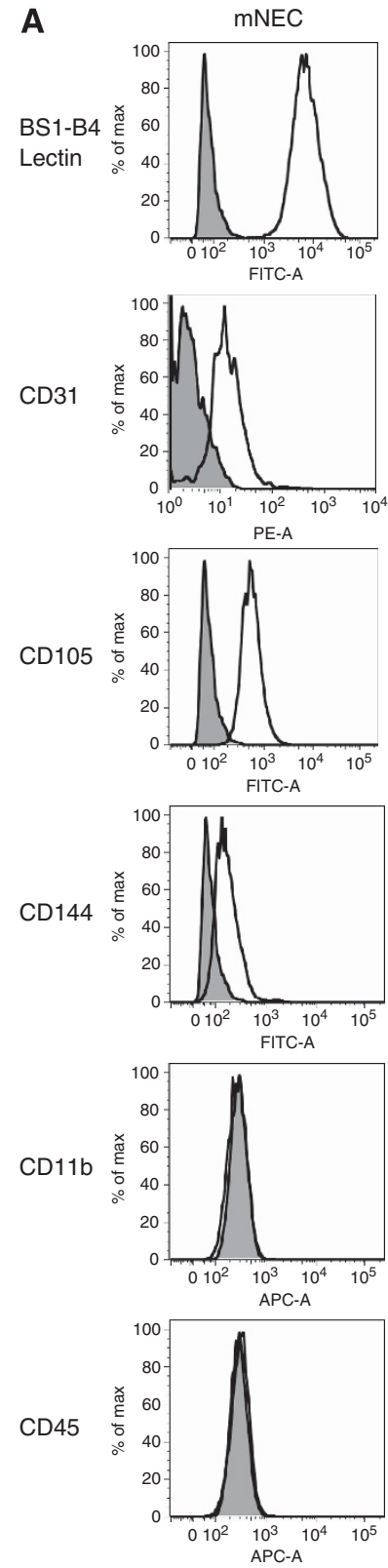
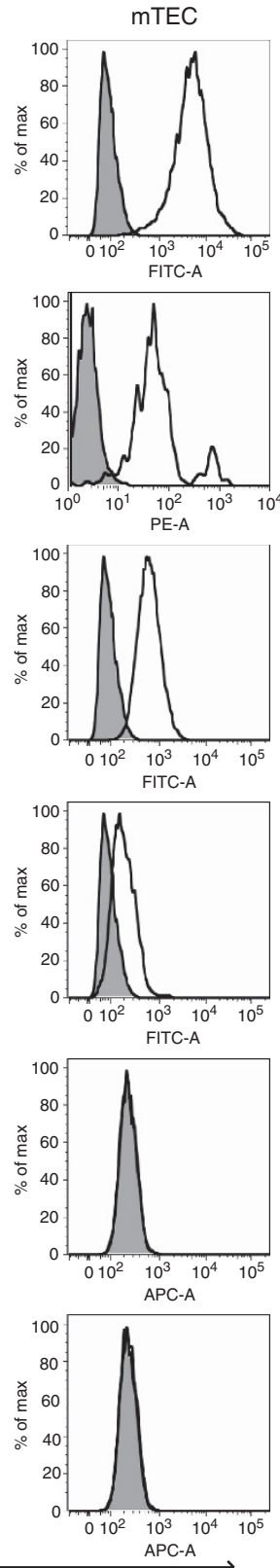

APC-A

Fluorescent intensity

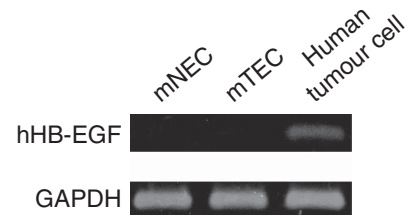

B

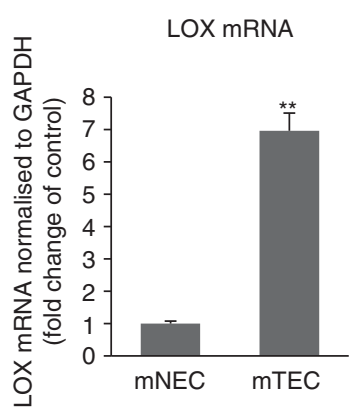

C
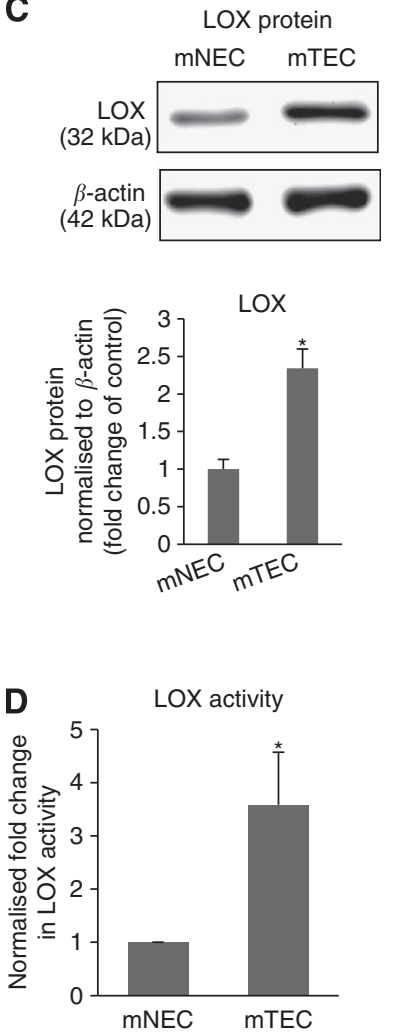

Figure 1. LOX expression in mTECs. (A) Characterisation of mTECs and mNECs. FACS analysis of BS1-B4 lectin binding and CD31, CD105 and CD144 expression (white areas). Isotype controls are shown as black areas. All ECs were negative for the monocyte marker CD11b and the hematopoietic marker CD45. Human HB-EGF mRNA expression was found in human tumour cells but not in mouse ECs. (B) LOX mRNA is upregulated in mTECs, as determined by $q P C R$. ${ }^{\star} P<<0.01$ vs mNECs (means \pm s.d.). (C) Western blotting revealed that LOX protein expression is upregulated in mTECs. ${ }^{*} P<0.05$ vs mNECs (means \pm s.d.). (D) LOX activity is higher in mTECs than in mNECs using Amplex Red. ${ }^{\star} P<0.05$ vs mNECs (means \pm s.d.).

and Froines, 1992). After confirming that BAPN inhibited LOX activity in TECs, ECs were treated with BAPN. BAPN significantly inhibited tube formation by TECs, but not by NECs (Supplementary Figure 4). Next, to assess the effects of LOX on tumour growth and metastasis in vivo, we established primary A375SM tumours in the subcutaneous tissues of nude mice and treated these mice with BAPN or vehicle. A375SM cells barely expressed LOX compared with MDA-MB-231 cells (positive control; Supplementary Figure 5). This agreed with previous reports for LOX expression in A375SM cells (Peinado et al, 2005; Payne et al, 2007). However, mTECs isolated from A375SM tumours exhibited high levels of LOX expression. Therefore, we hypothesised that BAPN administration would inhibit LOX activity in mTECs, but not in tumour cells. BAPN administration 


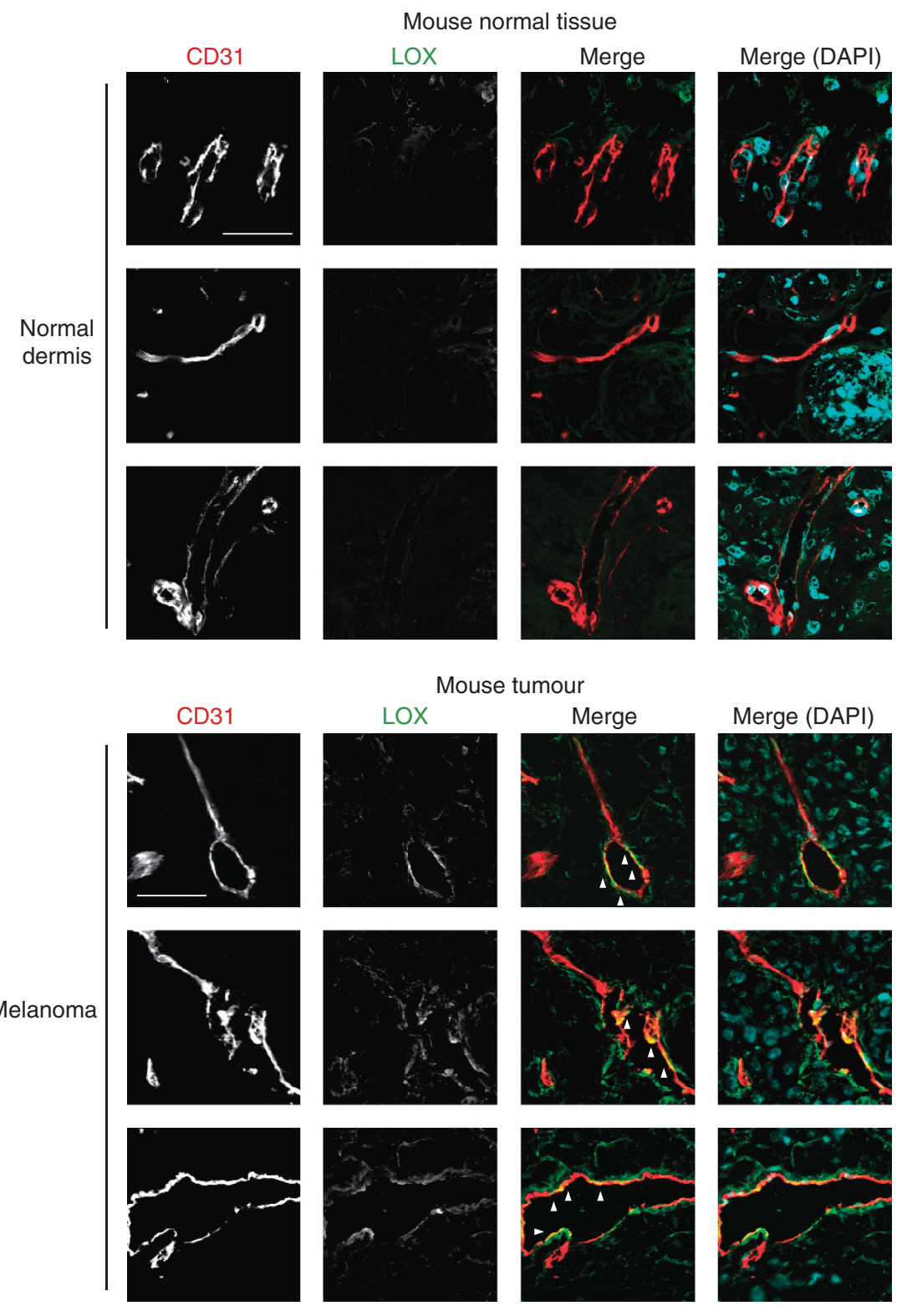

Figure 2. LOX is upregulated in tumour blood vessels in vivo. Immunostaining for the endothelial markers CD31 and LOX in tumours (A375SM) and in mouse normal tissues (dermal tissue). Merged staining (white arrow) shows co-localisation of LOX (green) and CD31 (red) in situ. DAPI stain: Nuclei. Scale bar $=50 \mu \mathrm{m}$.

did not affect tumour growth or tumour weight (Supplementary Figure 6A, B). However, BAPN significantly inhibited MVD (Figure 5A).

Next, we examined the effects of LOX inhibition on tumour metastasis. It is considered that circulating tumour cells (CTCs) are released into the bloodstream from tumours (Eccles and Welch, 2007) and that the numbers of CTCs are correlated with cancer patient outcomes (Hayes et al, 2006; de Bono et al, 2008). LOX expression levels were compared between two different TECs: currently used TECs isolated from highly metastatic tumours (A375SM) and TECs isolated from low-metastatic tumours (A375). LOX expression was significantly higher in high-metastatic tumour (A375SM)-derived TECs than in lowmetastatic tumour (A375)-derived TECs (Supplementary Figure 7).

To determine the effects of LOX inhibition on the intravasation of tumour cells into the systemic circulation, we counted CTCs by flow cytometry. The numbers of CTCs decreased in the
BAPN-treated group compared with the control (Figure 5B). We also evaluated the lungs of tumour-bearing mice using Picrosirius Red, which selectively stains cross-linked collagen fibrils when tissue sections are viewed under polarised light. The numbers of cross-linked collagen fibrils were increased in the lungs of tumour-bearing mice compared with the lungs of non-tumour mice. BAPN treatment significantly decreased collagen crosslinking in the lungs of tumour-bearing mice (Supplementary Figure 8).

Lung metastasis was verified by visualizing proliferating metastasised human tumour cells using anti-human Ki-67 staining. This antibody specifically detected human tumour cells in mouse lung tissue without background interference (Supplementary Figure 9). The number of metastatic colonies was significantly decreased in the BAPN-treated group compared with that in the control (Figure 5C). These results suggested that LOX inhibition suppressed tumour metastasis through an antiangiogenic effect. 
A

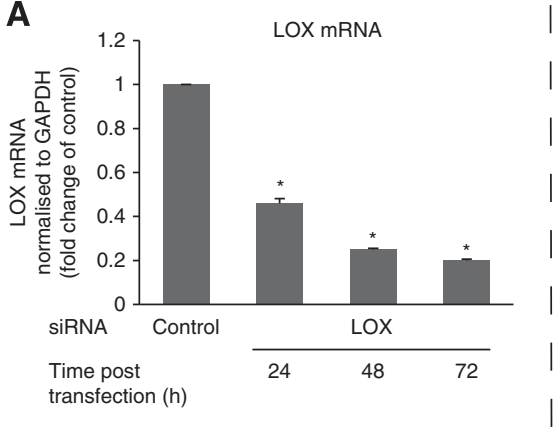

B

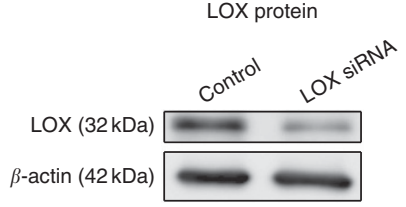

LOX
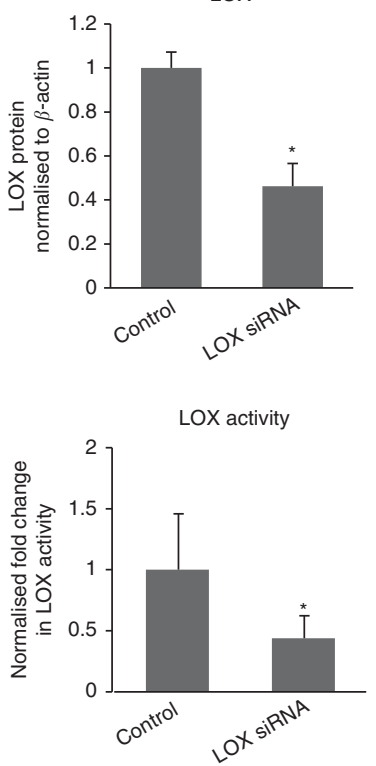

C

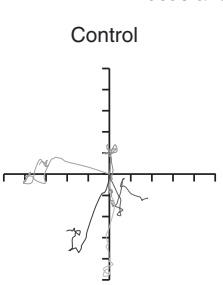

Locus analysis of mTEC

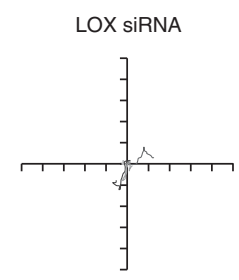

$-50 \mu \mathrm{m}$

Migration length
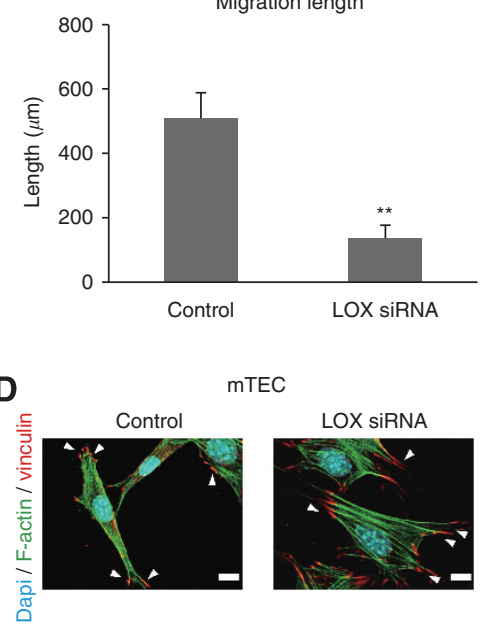

।

Ratio of cell length

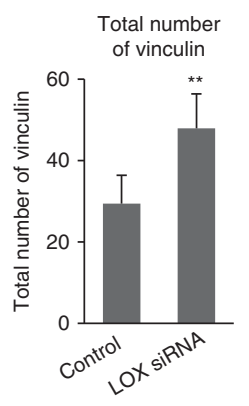

Figure 3. LOX knockdown by LOX siRNA in mTECs. (A) qPCR for LOX mRNA in mTECs transfected with siRNA. (B) LOX protein silencing was determined by western blotting at $48 \mathrm{~h}$ after transfection. LOX protein levels were normalised to that of $\beta$-actin and analysed using scanning densitometry using the NIH ImageJ software. ${ }^{*} P<0.05$ vs control (means \pm s.d.). LOX activity is decreased in LOX-knockdown $m T E C s$. ${ }^{\star} P<0.05$ vs control (means \pm s.d.). (C) Phenotypic changes in mTECs after LOX knockdown. Migration paths of siRNA-transfected mTECs. Each axis tick represents a distance of $50 \mu \mathrm{m}$. The migration distance of $\mathrm{mTEC}$ s transfected with siRNA was analysed. ${ }^{*} P<0.01$ vs control (means \pm s.d.; $n=10$ per group). (D) Immunostaining for actin organization in mTECs after staining with F-actin (green) and vinculin (red, white arrow). mTECs transfected with siRNA were examined by immunostaining. DAPI stain: Nuclei. Scale bar $=10 \mu \mathrm{m}$. Ratio of cell length to cell width. ${ }^{\star \star} P<0.01 \mathrm{vs}$ control (means \pm s.d.; $n=10$ per group). Vinculin was quantified in siRNA-transfected $m T E C s$. ${ }^{\star \star} P<0.01$ vs control (means \pm s.d.; $n=10$ per group).

LOX is upregulated in human TECs. Because renal cell carcinoma (RCC) is known to be angiogenic, we next isolated human TECs (hTECs) from human RCC tissues of six patients to investigate LOX expression in these cells. Human NECs (hNECs) were isolated from normal kidney tissues located away from the cancer tissues in these patients. The clinical backgrounds of these RCC specimens are shown in Table 1. Flow cytometry results for UEA1-lectin binding, CD31 and CD105 expression and negative CD45 expression showed that these isolated ECs were of high purity (Figure 6A).

LOX mRNA expression levels were significantly higher in hTECs than in hNECs for all paired cases (Figure 6B). These results suggested that LOX mRNA levels were elevated in hTECs and mTECs. To analyse LOX expression in hTECs in vivo, double immunostaining was used for the same frozen sections of human malignant tumours from the kidney, lung and colon using anti-LOX and anti-CD31 antibodies. LOX staining was strongly positive in tumour blood vessels but was negative in normal blood vessels (Figure 6C and Supplementary Figure 10), which suggested that LOX was upregulated in human renal TECs.

\section{DISCUSSION}

There were several new findings in this study. First, LOX expression was upregulated in mouse and human TECs both in vitro and in vivo. Second, LOX enhanced mTECs motility, possibly by downregulating vinculin expression. Third, LOX 
A FAK tyr 397 phosphorylation (western blotting)

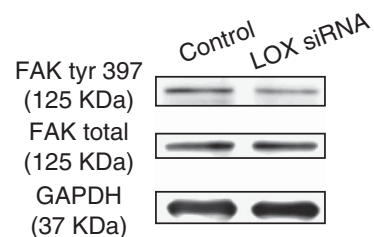

(37 KDa)

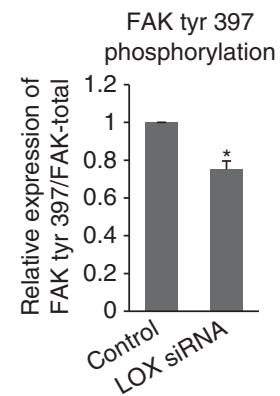

C
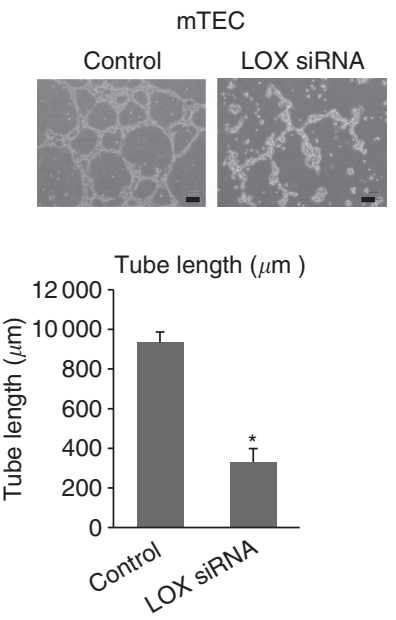

B

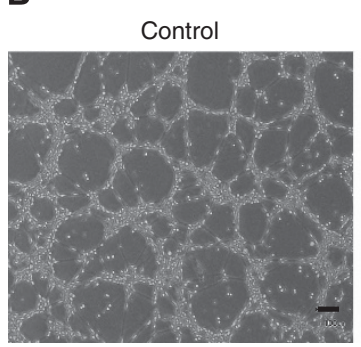

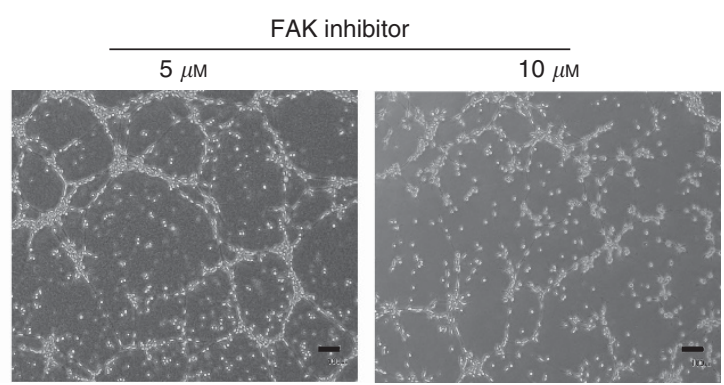

Figure 4. (A) Effects of LOX knockdown on FAK (Tyr 397) phosphorylation in mTECs after transfection with siRNA for $48 \mathrm{~h}$. GAPDH was used as an internal loading control. The levels of FAK phosphorylated at Tyr 397 were normalised to total FAK using the NIH Image $\mathrm{J}$ software. ${ }^{*} P<0.05$ vs control (means \pm s.d.). (B) Effects of FAK inhibition on mTEC tube formation. Scale bar $=100 \mu \mathrm{m}$. (C) Effects of LOX knockdown on mTEC tube formation. Tube formation was analysed using the $\mathrm{NIH}$ ImageJ software. ${ }^{\star} P<0.05$ vs control (means \pm s.d.). Diagram summarising tube lengths from three randomly selected light microscopic fields. Scale bar $=100 \mu \mathrm{m}$. ${ }^{\star} P<0.05$ vs corresponding control (means $\pm \mathrm{s}$.d.).

contributed to the pro-angiogenic phenotype of mTECs by promoting their tube formation. Fourth, a LOX inhibitor, BAPN, reduced tumour angiogenesis, the numbers of CTCs and pulmonary metastasis.

LOX is a collagen cross-linking enzyme that has a major role in remodelling the vascular extracellular matrix during angiogenesis. A recent report emphasised on the role of the LOX gene family in normal angiogenesis during early organ development (Bignon et al, 2011). Among LOX family members, LOXL2 is involved in angiogenesis (Baker et al, 2013). Although LOXL2 was expressed in our TECs, in a microarray analysis, the difference in LOXL2 expression between TECs and NECs was not significantly different compared with LOX. Thus, we investigated the role of LOX in TECs using siRNA. We confirmed that LOX knockdown did not affect LOXL2 expression and denied any off-target effects of LOX siRNA on LOXL2 (Supplementary Figure 11).

Tumour-derived LOX promotes angiogenesis (Baker et al, 2013). However, no previous studies have investigated LOX in TECs. Our results demonstrated that LOX was highly expressed in TECs. We did not determine the mechanism responsible for LOX upregulation in TECs. However, we speculate that VEGF induced by hypoxia may be one possibility because LOX expression is regulated by hypoxia-inducible factors (HIFs) and VEGF in tumour cells (Chronopoulos et al, 2010). The tumour vasculature is often leaky because of its immaturity, which creates high tissue pressure inside a tumour and tumour vessel shrinkage, which results in an insufficient blood supply. We previously reported that tumour blood vessels were also exposed to hypoxia and that VEGF was upregulated in TECs (Ohga et al, 2012). Furthermore, LOX was upregulated in NECs when they were exposed to hypoxic conditions or VEGF (Supplementary Figure 12).

We also found that cell migration was inhibited and cell morphology was spread out with increased vinculin expression after LOX knockdown in mTECs. This suggested that LOX may be an endogenous pro-angiogenic enzyme in TECs. Although the possibility of signalling between LOX and vinculin has not been determined, increased vinculin expression reportedly enhanced focal adhesion formation and reduced cell migration, as shown with LOX knockdown in mTECs (Coll et al, 1995; Humphries et al, 2007). Thus, vinculin regulation by LOX may have contributed to the enhanced cell migration by TECs.

BAPN is a known LOX inhibitor and is used to evaluate LOX enzymatic activity (Bondareva et al, 2009). BAPN treatment significantly decreased LOX activity in TECs and inhibited tube formation by TECs, but not by NECs, as shown in LOX siRNA experiments. This suggested that LOX enzymatic activity was also important for the angiogenic phenotype of TECs.

Many studies have shown that LOX was involved in human cancer cell invasiveness through FAK phosphorylation (Erler et al, 2006), which is important for cell migration (Tavora et al, 2010). A role for FAK in transmitting normal physiological angiogenic signals in ECs has also been suggested (Orr and 
Murphy-Ullrich, 2004). Thus, we speculated that FAK phosphorylation was involved in LOX-associated mTEC migration and tube formation. LOX promoted FAK (Tyr 397) phosphorylation in mTECs, which suggested that LOX had an important role in the motility and pro-angiogenic phenotype of mTECs at least through FAK (Tyr 397) phosphorylation.
A

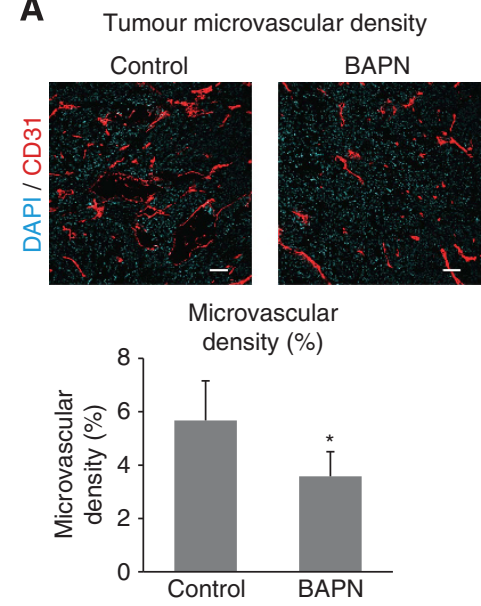

B The number of

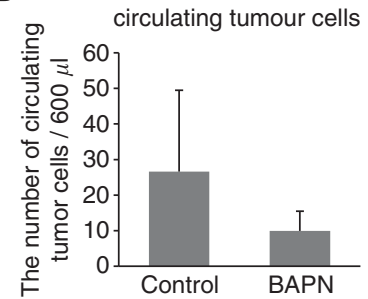

C Micrometastasis in the mouse lung

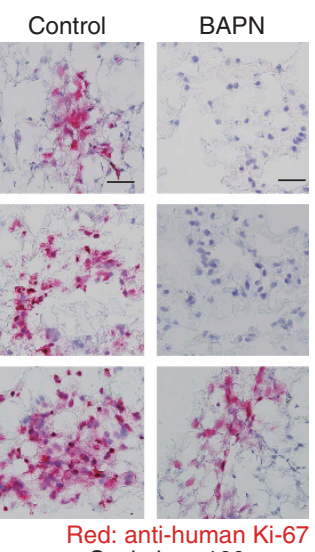

Scale bar: $100 \mu \mathrm{m}$

Number of tumour colonies in the lung

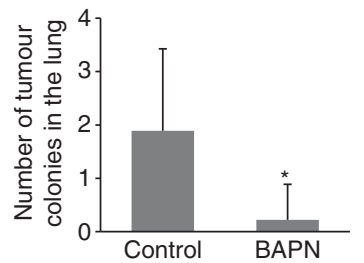

Figure 5. In vivo experiment using BAPN. (A) Vascularisation within a primary A375SM tumour was assessed by CD31 immunostaining. Scale $\mathrm{bar}=100 \mu \mathrm{m}$. For microvascular density (MVD) analysis, the areas of vessels in CD31-stained sections were determined using the Image J software. ${ }^{*} P<0.05$ vs control (means \pm s.d.; $n=15$ per group). (B) BAPN treatment decreased the numbers of circulating tumour cells (CTCs) in tumour-bearing mice. (means \pm s.d.; $n=3$ per group). (C) Metastatic colonies in lungs were visualised by staining with anti-human Ki-67. BAPN treatment suppressed tumour metastasis. Scale bar $=100 \mu \mathrm{m}$. The numbers of tumour colonies in lungs, defined as $>20$ anti-human Ki-67 cells/area, decreased in BAPN-treated mice. ${ }^{*} P<0.05$ vs control (means \pm s.d.; $n=9$ per group).
Regarding LOX expression in other cell types in tumour tissues, LOX mRNA was very weakly expressed in CD31-negative stromal cells in vitro, including fibroblasts (Supplementary Figure 13). In addition, by imunohistchemical analysis, LOX was not expressed in tumour cells or in CD31-negative stromal cells in vivo, which suggested that LOX was only highly expressed in TECs in our tumour model.

A375SM cells used in our in vivo experiments barely expressed LOX mRNA, whereas TECs exhibited significantly high levels of LOX expression. Thus, BAPN appeared to primarily affect TECs but not tumour cells in this in vivo model. Furthermore, BAPN suppressed pulmonary metastasis along with reduced numbers of CTCs through its angiogenesis inhibition activity. Several studies have also shown that tumour growth was not inhibited by BAPN, although it reduced tumour metastasis (Bondareva et al, 2009). It has also been observed that even when tumour growth did not change, tumour metastasis was sometimes dramatically altered. In these cases, tumour angiogenesis had a key role in metastasis. Because tumour blood vessels are important for tumour metastasis (Folkman, 1995), inhibiting tumour angiogenesis could be one mechanism for suppressing metastasis by LOX inhibition.

Furthermore, LOX is critical for pre-metastatic niche formation and its inhibition prevents metastatic tumour growth through decreased recruitment of bone marrow-derived cells (Erler et al, 2009). However, blood vessels provide an outlet for tumour cells to leave their tumour nests. Thus, tumour cell entry into the blood may be prevented by suppressing tumour angiogenesis through LOX inhibition.

It is known that the primary function of LOX is to promote the covalent cross-linking of collagens and/or elastin in the extracellular matrix (ECM), which mediates tumour malignant transformation (Levental et al, 2009) and the formation of premetastatic niches (Erler et al, 2009). Thus, TEC-derived LOX might be involved in pre-metastatic niche formation.

Our results showed that tumour intravasation and pulmonary metastasis were decreased when BAPN reduced the tumour microvasculature. In addition, cross-linked collagen fibrils were increased in the lungs of tumour-bearing mice but were decreased when mice were treated with BAPN. This suggested that LOX secreted by TECs in primary tumours might regulate collagen remodelling in the lungs. It has been reported that collagen crosslinking was related to metastatic niche formation. TEC-derived LOX may be another mechanism for tumour metastasis by inducing collagen cross-linking at metastatic sites. Regarding the relationship between lung metastasis and TEC-derived LOX, we have not determined whether this was due to ECM modifications or some other mechanism besides enhanced tumour angiogenesis. Additional studies will be needed to unravel this mechanism.

Table 1. Background of renal cell carcinoma (RCC) samples

\begin{tabular}{|c|c|c|c|c|c|}
\hline Sample no. & $M / F$ & Age (yr) & TNM $^{a}$ & Subtype & Grade $^{b}$, INF, v \\
\hline 1 & $\mathrm{~F}$ & 53 & $\mathrm{T1a}, \mathrm{Nx}, \mathrm{MO}$ & Clear cell & $\mathrm{G} 3, \mathrm{INFa}, \mathrm{v}(-)$ \\
\hline 2 & $\mathrm{~F}$ & 66 & $\mathrm{~T} 3 \mathrm{~b}, \mathrm{~N} 1, \mathrm{M} 1$ & Clear cell & $\mathrm{G} 3, \mathrm{INFb}, \mathrm{v}(+)$ \\
\hline 3 & $\mathrm{M}$ & 76 & T3b, NO, MO & Clear cell & $\mathrm{G} 2, \mathrm{INFa}, \mathrm{v}(+)$ \\
\hline 4 & $\mathrm{M}$ & 65 & T3a, N0, M1 & Clear cell & $\mathrm{G} 3, \mathrm{INFb}, \mathrm{v}(+)$ \\
\hline 5 & $\mathrm{~F}$ & 50 & $\mathrm{~T} 1 \mathrm{~b}, \mathrm{Nx}, \mathrm{MO}$ & Clear cell & $\mathrm{G} 2, \mathrm{INFa}, \mathrm{v}(-)$ \\
\hline 6 & M & 65 & $\mathrm{~T} 1 \mathrm{a}, \mathrm{Nx}, \mathrm{M} 0$ & Clear cell & $\mathrm{G} 1, \mathrm{INFa}, \mathrm{v}(-)$ \\
\hline
\end{tabular}


A
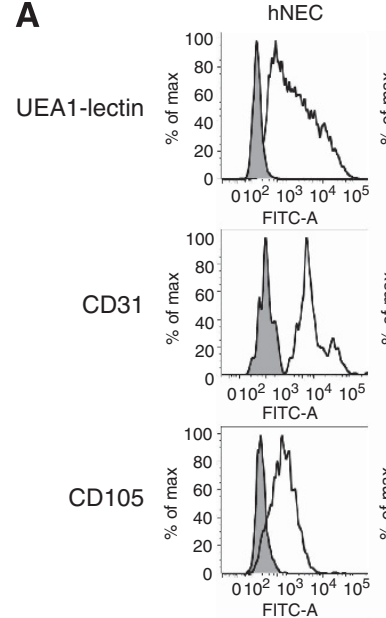

CD45
B
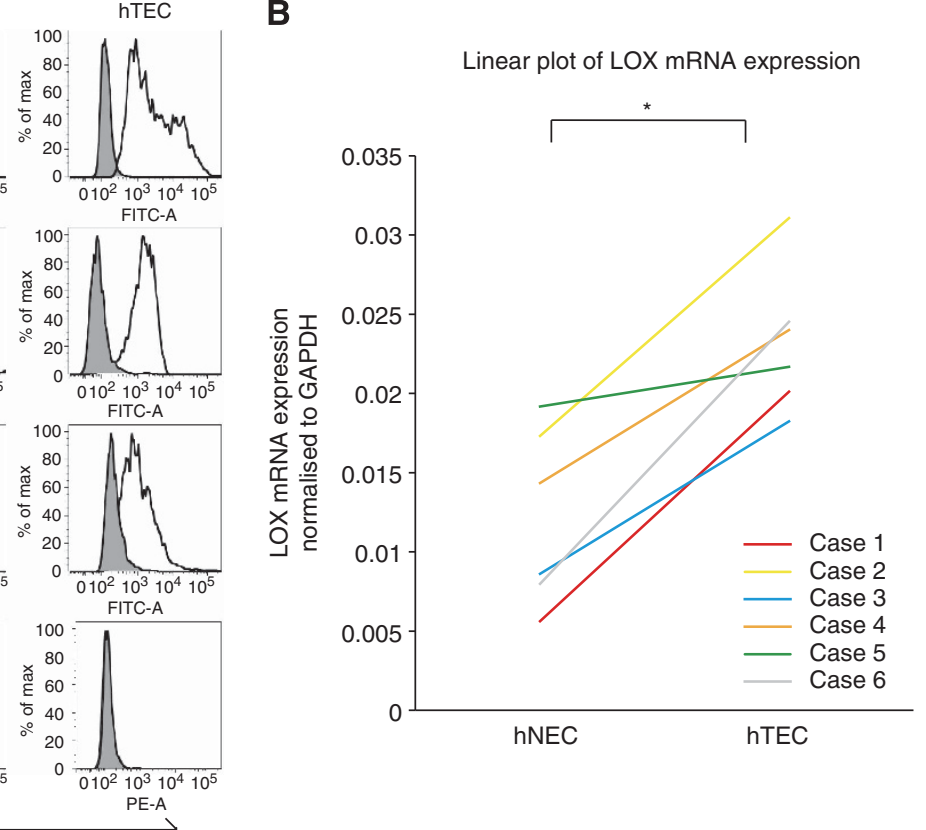

Fluorescent intensity
C
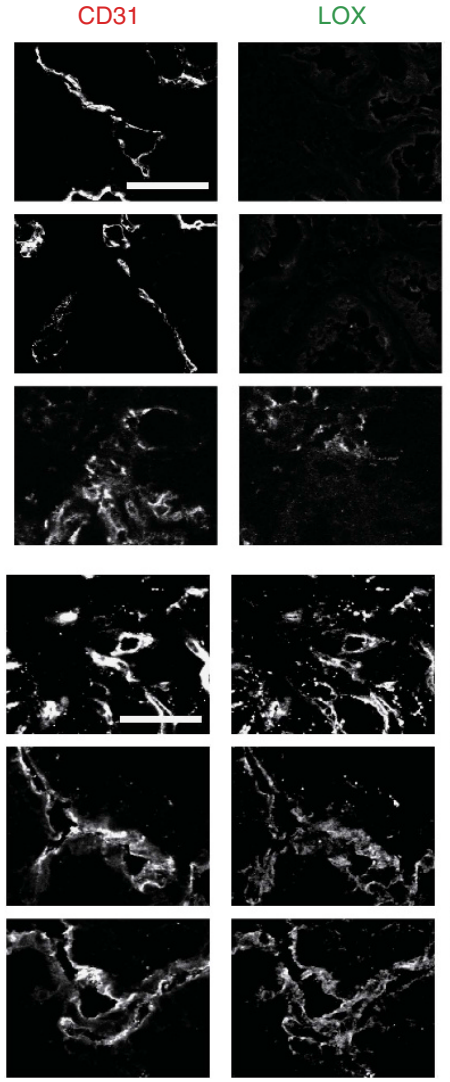

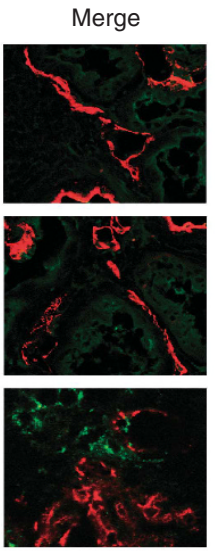

Merge (DAPI)
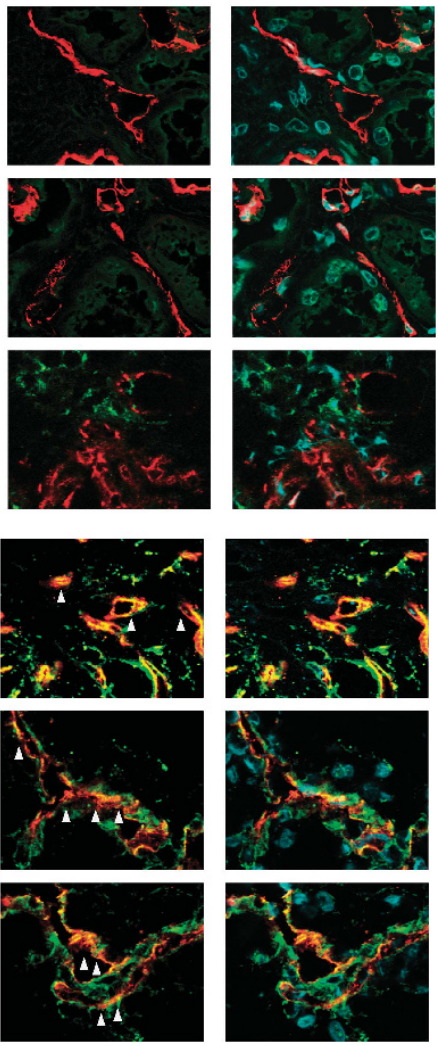

Figure 6. LOX expression in hTECs from human RCC. (A) FACS analysis of hTECs showing UEA1-lectin binding, expression of the endothelial markers CD31 and CD105 and no CD45 expression (right line). Left line is the isotype control. (B) qPCR to confirm that LOX mRNA was highly expressed in all six hTECs compared with corresponding hNECs. Linear plot for LOX mRNA expression in hNECs and hTECs for six case-matched samples. Each point is the average of three independent qPCR results. ${ }^{*} P<0.05$ vs control; paired $t$-test (means \pm s.d.; $n=6$ per group). (C) Tumour blood vessels in human RCC were double stained with anti-CD31 and anti-LOX antibodies. LOX protein was highly expressed in the blood vessels of human RCC. Scale bar $=50 \mu \mathrm{m}$.

Finally, we demonstrated that LOX was highly expressed in mouse and human TECs. Thus, for the first time, we have shown that LOX activates the pro-angiogenic phenotype in TECs and that its inhibition may be effective for suppressing tumour metastasis. LOX may be a TEC-specific marker and a possible candidate for vascular targeted cancer therapy. 


\section{ACKNOWLEDGEMENTS}

We thank Dr IJ Fidler for providing the A375SM super-metastatic human malignant melanoma cell line. We also thank Dr Aya Yanagawa, Ms Tomomi Takahashi, Mrs Midori Muranaka, Ms H Omura, Mr Y Sadamoto, Ms C Torii and Ms Y Suzuki for their technical assistance. This work was supported by Grants-in-Aid for Scientific Research from the Ministry of Education, Science and Culture of Japan (K Hida, Y Hida and N Ohga).

\section{REFERENCES}

Akino T, Hida K, Hida Y, Tsuchiya K, Freedman D, Muraki C, Ohga N, Matsuda K, Akiyama K, Harabayashi T, Shinohara N, Nonomura K, Klagsbrun M, Shindoh M (2009) Cytogenetic abnormalities of tumour-associated endothelial cells in human malignant tumors. Am J Pathol 175: 2657-2667.

Akiyama K, Ohga N, Hida Y, Kawamoto T, Sadamoto Y, Ishikawa S, Maishi N, Akino T, Kondoh M, Matsuda A, Inoue N, Shindoh M, Hida K (2012) Tumor endothelial cells acquire drug resistance by MDR1 up-regulation via VEGF signaling in tumor microenvironment. Am J Pathol 180: 1283-1293.

Amin DN, Hida K, Bielenberg DR, Klagsbrun M (2006) Tumor endothelial cells express epidermal growth factor receptor (EGFR) but not ErbB3 and are responsive to EGF and to EGFR kinase inhibitors. Cancer Res 66 2173-2180.

Baker AM, Bird D, Welti JC, Gourlaouen M, Lang G, Murray GI, Reynolds AR, Cox TR, Erler JT (2013) Lysyl oxidase plays a critical role in endothelial cell stimulation to drive tumor angiogenesis. Cancer Res 73: 583-594.

Bignon M, Pichol-Thievend C, Hardouin J, Malbouyres M, Brechot N, Nasciutti L, Barret A, Teillon J, Guillon E, Etienne E, Caron M, Joubert-Caron R, Monnot C, Ruggiero F, Muller L, Germain S (2011) Lysyl oxidase-like protein-2 regulates sprouting angiogenesis and type IV collagen assembly in the endothelial basement membrane. Blood 118: 3979-3989.

Bondareva A, Downey CM, Ayres F, Liu W, Boyd SK, Hallgrimsson B, Jirik FR (2009) The lysyl oxidase inhibitor, beta-aminopropionitrile, diminishes the metastatic colonization potential of circulating breast cancer cells. PLoS One 4: e5620.

Bussolati B, Deambrosis I, Russo S, Deregibus MC, Camussi G (2003) Altered angiogenesis and survival in human tumor-derived endothelial cells. FASEB J 17: 1159-1161.

Chronopoulos A, Tang A, Beglova E, Trackman PC, Roy S (2010) High glucose increases lysyl oxidase expression and activity in retinal endothelial cells: mechanism for compromised extracellular matrix barrier function. Diabetes 59: 3159-3166.

Coll JL, Ben-Ze'ev A, Ezzell RM, Rodriguez Fernandez JL, Baribault H, Oshima RG, Adamson ED (1995) Targeted disruption of vinculin genes in F9 and embryonic stem cells changes cell morphology, adhesion, and locomotion. Proc Natl Acad Sci USA 92: 9161-9165.

de Bono JS, Scher HI, Montgomery RB, Parker C, Miller MC, Tissing H, Doyle GV, Terstappen LW, Pienta KJ, Raghavan D (2008) Circulating tumor cells predict survival benefit from treatment in metastatic castration-resistant prostate cancer. Clin Cancer Res 14: 6302-6309.

Eccles SA, Welch DR (2007) Metastasis: recent discoveries and novel treatment strategies. Lancet 369: 1742-1757.

Erler JT, Bennewith KL, Cox TR, Lang G, Bird D, Koong A, Le QT, Giaccia AJ (2009) Hypoxia-induced lysyl oxidase is a critical mediator of bone marrow cell recruitment to form the premetastatic niche. Cancer Cell 15: $35-44$.

Erler JT, Bennewith KL, Nicolau M, Dornhofer N, Kong C, Le QT, Chi JT, Jeffrey SS, Giaccia AJ (2006) Lysyl oxidase is essential for hypoxia-induced metastasis. Nature 440: 1222-1226.

Folkman J (1971) Tumor angiogenesis: therapeutic implications. N Engl J Med 285: $1182-1186$.

Folkman J (1995) Angiogenesis in cancer, vascular, rheumatoid and other disease. Nat Med 1: 27-31.

Hayes DF, Cristofanilli M, Budd GT, Ellis MJ, Stopeck A, Miller MC, Matera J, Allard WJ, Doyle GV, Terstappen LW (2006) Circulating tumor cells at each follow-up time point during therapy of metastatic breast cancer patients predict progression-free and overall survival. Clin Cancer Res 12: 4218-4224.

Hida K, Hida Y, Amin DN, Flint AF, Panigrahy D, Morton CC, Klagsbrun M (2004) Tumor-associated endothelial cells with cytogenetic abnormalities. Cancer Res 64: 8249-8255.

Hida K, Klagsbrun M (2005) A new perspective on tumor endothelial cells: unexpected chromosome and centrosome abnormalities. Cancer Res 65: 2507-2510.

Humphries JD, Wang P, Streuli C, Geiger B, Humphries MJ, Ballestrem C (2007) Vinculin controls focal adhesion formation by direct interactions with talin and actin. J Cell Biol 179: 1043-1057.

Kawamoto T, Ohga N, Akiyama K, Hirata N, Kitahara S, Maishi N, Osawa T, Yamamoto K, Kondoh M, Shindoh M, Hida Y, Hida K (2012) Tumorderived microvesicles induce proangiogenic phenotype in endothelial cells via endocytosis. PLoS One 7: e34045.

Kirschmann DA, Seftor EA, Fong SF, Nieva DR, Sullivan CM, Edwards EM, Sommer P, Csiszar K, Hendrix MJ (2002) A molecular role for lysyl oxidase in breast cancer invasion. Cancer Res 62: 4478-4483.

Kurosu T, Ohga N, Hida Y, Maishi N, Akiyama K, Kakuguchi W, Kuroshima T, Kondo M, Akino T, Totsuka Y, Shindoh M, Higashino F, Hida K (2011) HuR keeps an angiogenic switch on by stabilising mRNA of VEGF and COX-2 in tumour endothelium. Br J Cancer 104: 819-829.

Levental KR, Yu H, Kass L, Lakins JN, Egeblad M, Erler JT, Fong SF, Csiszar K, Giaccia A, Weninger W, Yamauchi M, Gasser DL, Weaver VM (2009) Matrix crosslinking forces tumor progression by enhancing integrin signaling. Cell 139: 891-906.

Lucero HA, Kagan HM (2006) Lysyl oxidase: an oxidative enzyme and effector of cell function. Cell Mol Life Sci 63: 2304-2316.

Maishi N, Ohga N, Hida Y, Akiyama K, Kitayama K, Osawa T, Onodera Y, Shinohara N, Nonomura K, Shindoh M, Hida K (2012) CXCR7: a novel tumor endothelial marker in renal cell carcinoma. Pathol Int 62: 309-317.

Matsuda K, Ohga N, Hida Y, Muraki C, Tsuchiya K, Kurosu T, Akino T, Shih SC, Totsuka Y, Klagsbrun M, Shindoh M, Hida K (2010) Isolated tumor endothelial cells maintain specific character during long-term culture. Biochem Biophys Res Commun 394: 947-954.

McCarthy M (2003) Antiangiogenesis drug promising for metastatic colorectal cancer. Lancet 361: 1959.

Muraki C, Ohga N, Hida Y, Nishihara H, Kato Y, Tsuchiya K, Matsuda K, Totsuka Y, Shindoh M, Hida K (2011) Cyclooxygenase-2 inhibition causes antiangiogenic effects on tumor endothelial and vascular progenitor cells. Int J Cancer 130: 59-70.

Ohga N, Hida K, Hida Y, Muraki C, Tsuchiya K, Matsuda K, Ohiro Y, Totsuka Y, Shindoh M (2009) Inhibitory effects of epigallocatechin-3 gallate, a polyphenol in green tea, on tumor-associated endothelial cells and endothelial progenitor cells. Cancer Sci 100: 1963-1970.

Ohga N, Ishikawa S, Maishi N, Akiyama K, Hida Y, Kawamoto T, Sadamoto Y, Osawa T, Yamamoto K, Kondoh M, Ohmura H, Shinohara N, Nonomura K, Shindoh M, Hida K (2012) Heterogeneity of tumor endothelial cells: comparison between tumor endothelial cells isolated from high- and low-metastatic tumors. Am J Pathol 180: 1294-1307.

Orr AW, Murphy-Ullrich JE (2004) Regulation of endothelial cell function BY FAK and PYK2. Front Biosci 9: 1254-1266.

Osawa T, Ohga N, Hida Y, Kitayama K, Akiyama K, Onodera Y, Fujie M, Shinohara N, Shindoh M, Nonomura K, Hida K (2012) Prostacyclin receptor in tumor endothelial cells promotes angiogenesis in an autocrine manner. Cancer Sci 103: 1038-1044.

Panchenko MV, Stetler-Stevenson WG, Trubetskoy OV, Gacheru SN, Kagan HM (1996) Metalloproteinase activity secreted by fibrogenic cells in the processing of prolysyl oxidase. Potential role of procollagen C-proteinase. J Biol Chem 271: 7113-7119.

Payne SL, Hendrix MJ, Kirschmann DA (2007) Paradoxical roles for lysyl oxidases in cancer-a prospect. J Cell Biochem 101: 1338-1354.

Peinado H, Del Carmen Iglesias-de la Cruz M, Olmeda D, Csiszar K, Fong KS, Vega S, Nieto MA, Cano A, Portillo F (2005) A molecular role for lysyl oxidase-like 2 enzyme in snail regulation and tumor progression. EMBO J 24: 3446-3458.

Randall LM, Monk BJ (2010) Bevacizumab toxicities and their management in ovarian cancer. Gynecol Oncol 117: 497-504.

Rodriguez HM, Vaysberg M, Mikels A, McCauley S, Velayo AC, Garcia C, Smith V (2010) Modulation of lysyl oxidase-like 
2 enzymatic activity by an allosteric antibody inhibitor. J Biol Chem 285: 20964-20974.

Small JV (1981) Organization of actin in the leading edge of cultured cells: influence of osmium tetroxide and dehydration on the ultrastructure of actin meshworks. J Cell Biol 91: 695-705.

St Croix B, Rago C, Velculescu V, Traverso G, Romans KE, Montgomery E, Lal A, Riggins GJ, Lengauer C, Vogelstein B, Kinzler KW (2000) Genes expressed in human tumor endothelium. Science 289: 1197-1202.

Tavora B, Batista S, Reynolds LE, Jadeja S, Robinson S, Kostourou V, Hart I, Fruttiger M, Parsons M, Hodivala-Dilke KM (2010) Endothelial FAK is required for tumour angiogenesis. EMBO Mol Med 2: 516-528.

Trackman PC, Bedell-Hogan D, Tang J, Kagan HM (1992) Post-translational glycosylation and proteolytic processing of a lysyl oxidase precursor. J Biol Chem 267: 8666-8671.

Tsuchiya K, Hida K, Hida Y, Muraki C, Ohga N, Akino T, Kondo T, Miseki T, Nakagawa K, Shindoh M, Harabayashi T, Shinohara N, Nonomura K, Kobayashi M (2010) Adrenomedullin antagonist suppresses tumor formation in renal cell carcinoma through inhibitory effects on tumor endothelial cells and endothelial progenitor mobilization. Int J Oncol 36: 1379-1386.

Wilmarth KR, Froines JR (1992) In vitro and in vivo inhibition of lysyl oxidase by aminopropionitriles. J Toxicol Environ Health 37: 411-423.

Yamamoto K, Ohga N, Hida Y, Maishi N, Kawamoto T, Kitayama K, Akiyama K, Osawa T, Kondoh M, Matsuda K, Onodera Y, Fujie M, Kaga K, Hirano S, Shinohara N, Shindoh M, Hida K (2012) Biglycan is a specific marker and an autocrine angiogenic factor of tumour endothelial cells. Br J Cancer 106: 1214-1223.

This work is published under the standard license to publish agreement. After 12 months the work will become freely available and the license terms will switch to a Creative Commons AttributionNonCommercial-Share Alike 3.0 Unported License.

Supplementary Information accompanies this paper on British Journal of Cancer website (http://www.nature.com/bjc) 